\title{
SCORE 1.0 - Um Instrumento para Autoavaliação de Criatividade voltado ao Ensino de Computação
}

\author{
Géssica Lehmkuhl $^{1}$, Christiane Gresse von Wangenheim ${ }^{1}$, Lúcia Martins-Pacheco ${ }^{1}$, \\ Adriano F. Borgatto ${ }^{1}$, Nathalia da Cruz Alves ${ }^{1}$ \\ ${ }^{1}$ Departamento de Informática e Estatística - Universidade Federal de Santa Catarina \\ (UFSC) \\ Campus Trindade - 88040-900 - Florianópolis - SC - Brasil \\ gessica.lehmkuhl@grad.ufsc.br, \{c.wangenheim, lucia.pacheco, \\ adriano.borgatto\}@ufsc.br, nathalia.alves@posgrad.ufsc.br
}

\begin{abstract}
Computing education can also contribute to the development of creativity in K-12. However, despite the existence of some creativity assessments, there is a lack of models for the context of computing education in K-12. Therefore, we present an instrument for the self-assessment of creativity in this context systematically developed based on literature. An initial evaluation of the instrument indicates good reliability (Cronbach alfa $=0.961$ ) and validity. Thus, the results of this research can be used to guide the development of students' creativity as well as for the design of instructional units aiming at this learning objective.
\end{abstract}

Resumo. O ensino de computação na Educação Básica pode também contribuir no desenvolvimento da criatividade. Mesmo existindo diversos modelos de avaliação da criatividade na literatura, constata-se uma carência de modelos voltados ao ensino de computação. Assim, neste contexto, apresentamos um instrumento de autoavaliação da criatividade desenvolvido sistematicamente a partir da literatura. Uma avaliação inicial do instrumento, indica uma boa confiabilidade (Cronbach alfa $=0,961)$ e validade. Os resultados desta pesquisa podem ser utilizados para guiar o desenvolvimento da criatividade dos estudantes quanto ao design de unidades instrucionais visando esse objetivo de aprendizagem.

\section{Introdução}

A criatividade e o seu desenvolvimento na formação dos estudantes são muito importantes, pois possibilita a promoção de novas soluções no âmbito econômico, social e de inovações tecnológicas [Richardson e Mishra 2018][Pásztor et al. 2015]. No processo educacional ela é considerada uma habilidade essencial no século XXI, para a profissão e para a vida pessoal [Binkley et al. 2010][Nakano 2020][Mioto et al. 2019][Cavallo et al. 2016]. Ser criativo engloba habilidades cognitivas, como a capacidade de identificar problemas, gerar ideias por meio de pensamentos originais e a resolução de problemas [Lai e Viering 2012].

Apesar da multiplicidade de definições da criatividade [Nakano 2020], há alguns pontos convergentes. Por exemplo, a caracterização de que algo criativo é algo útil, novo, inesperado e original [Pásztor et al. 2015], [Hennessey e Amabile 1988]. Ser algo útil está associado a uma solução adequada para a tarefa ou problema proposto. Outro aspecto que costuma ser destacado é o chamado pensamento divergente, como habilidade de gerar o maior número possível de soluções para um problema [Kupers et al. 2018]. Há muitas 
IX Congresso Brasileiro de Informática na Educação (CBIE 2020)

Anais do XXXI Simpósio Brasileiro de Informática na Educação (SBIE 2020)

propostas teóricas de caracterizar o construto criatividade, entre elas o modelo proposto por Guilford (1950), que caracteriza criatividade como:

- Fluência: a capacidade de gerar muitas ideias, o que libera a criatividade.

- Flexibilidade: a capacidade de analisar uma situação de um ângulo diferente, por meio da combinação de diferentes lugares, pessoas, sentidos e períodos.

- Originalidade: a capacidade de gerar produtos únicos ou incomuns.

- Elaboração: a capacidade de envolver detalhes, embelezar e concluir algo criativo.

O pensamento divergente pode ser considerado um tipo de pensamento criativo e, apesar de não serem a mesma coisa, ambos levam a ideias e soluções originais [Runco e Acar, 2012].

$\mathrm{Na}$ abordagem 4P's (process, person, product and press) de Rhodes (1961), a criatividade se apresenta em diferentes perspectivas: processo (ações em tempo real), pessoa (características pessoais, atitudes e comportamentos), produto (o que torna um produto criativo) e ambiente criativo (características de ambientes que promovem a criatividade).

Paralelamente a complexidade da caracterização teórica do construto criatividade, está a dificuldade de observá-lo, avaliá-lo e medi-lo. Nesse sentido, testes, inventários, julgamento dos produtos criados, autorrelatos de atividades criativas [Nakano 2020], dentre outros instrumentos, vêm sendo desenvolvidos e pesquisados. O TTCT (Torrance Tests of Creative Thinking) [Torrance 1966] é um dos testes mais conhecidos. Esse teste associa das características cognitivas propostas por Guilford (1956) e as características emocionais como expressão da emoção, fantasia e movimento, dentre outras. Outros instrumentos incluem elementos como pensamento divergente, pensamento analítico, flexibilidade mental, pensamento associativo, tolerância à ambiguidade, imaginação e capacidade inventiva [Nakano 2020]. De acordo com a autora, os métodos de avaliação da criatividade também podem ser classificados como objetivos e subjetivos. Os dois tipos são considerados válidos e podem ser utilizados em conjunto. O TTCT é considerado objetivo, já os subjetivos são os de julgamento de produtos por juízes, indicações realizadas por pares e a autoavaliação.

Com relação a autoavaliação da criatividade, Kaufman (2019) considera que essa é uma opção frequentemente usada nas pesquisas empíricas, pois normalmente os instrumentos são de uso gratuito, rápida administração e fácil pontuação. Para ele, a significância desse tipo de avaliação depende do tipo de instrumento, de sua relação com as medidas baseadas em desempenho e com o conjunto de conceitos subjacentes que estão sendo medidos. As limitações desse tipo de avaliação estão associadas a questões subjetivas dos respondentes, como respostas idealizadas por eles como desejáveis, inverdades ou exageros para aparentar ser melhor, também relacionadas ao fato de muitas pessoas não perceberem suas próprias habilidades criativas (subestimando ou superestimando) ou ao conceito pessoal de criatividade. Para Kaufman (2019) a credibilidade da autoavaliação da criatividade depende do seu uso e pode apresentar uma boa aproximação dos testes consolidados baseados em medidas de desempenho. Igualmente, a autoavaliação da criatividade pode fazer uma estimativa de como algo impacta na forma como a pessoa se sente em relação a sua criatividade e, em muitos casos, representar a melhor medida possível no que se refere a examinar as crenças pessoais e os insights sobre a concepção própria de criatividade.

Instrumentos para a avaliação da criatividade são fundamentais para que se consiga promover essa habilidade nos estudantes, permitindo a aferição de suas expressões criativas. As demandas educacionais, em especial aquelas relacionadas às tecnologias da informação e comunicação a as demais habilidade do século XXI, tem na promoção e avaliação da 
criatividade importantes aliados [Bordini et al. 2017]. O desenvolvimento de criatividade desde a Educação Básica permite a formação de pessoas competentes em originalidade, flexibilidade, iniciativa e confiança possibilitando que enfrentem os obstáculos que se apresentarão na vida escolar e cotidiana, além de oferecer ferramentas para inovação [Sousa Filho 2011]. Nesse sentido, Shell et al. (2012) propôs que o aprendizado do pensamento computacional pode ser aperfeiçoado por meio da união entre o pensamento computacional e o pensamento criativo, chamado de criatividade computacional. Com a criatividade computacional, alunos conseguem expandir o conhecimento e as habilidades para aplicar na resolução de um problema e em outras disciplinas. São habilidades que se complementam, da mesma forma que o pensamento computacional amplia a habilidade de resolver um problema criativamente, a criatividade aperfeiçoa o desenvolvimento do pensamento computacional.

Assim, buscando contribuir com a promoção da criatividade, este artigo apresenta o instrumento de autoavaliação da criatividade SCORE 1.0, no contexto do ensino de computação na Educação Básica. Esse instrumento visa contribuir para a autopercepção dos estudantes de aspectos relevantes para o desenvolvimento da criatividade.

\section{Trabalhos Relacionados}

Como resultado de um mapeamento sistemático [Lehmkuhl 2020] foram encontrados somente dez modelos voltado a autoavaliação de criatividade no contexto educacional de interesse, conforme apresentado na Tabela 1.

Tabela 1. Modelos de avaliação da criatividade encontrados no MSL

\begin{tabular}{|c|c|}
\hline Nome do Modelo & Referência \\
\hline $\begin{array}{l}\text { CT - Abedi-Schumacher Creativity } \\
\text { Test }\end{array}$ & $\begin{array}{l}\text { Auzmendi, E. et al. (2016) "Reliability and Validity of a Newly Constructed Multiple-Choice } \\
\text { Creativity Instrument", Creativity Research Journal, 9(1), 89-95. }\end{array}$ \\
\hline Não informado & $\begin{array}{l}\text { Hass, R. W., Burke, S. (2016) "Implicit theories of creativity are differentially categorized by } \\
\text { perspective and exemplar domain", Thinking Skills and Creativity, 19, 219-231. }\end{array}$ \\
\hline $\begin{array}{l}\text { K-DOCS - Kaufman Domains of } \\
\text { Creativity Scale }\end{array}$ & $\begin{array}{l}\text { Kaufman, J. C. (2012) Counting the Muses: Development of the Kaufman Domains of Creativity } \\
\text { Scale (K-DOCS). Psychology of Aesthetics Creativity and the Arts. vol. 6(4), p. 298-308. }\end{array}$ \\
\hline Student Engagement Survey & $\begin{array}{l}\text { McKlin, T. et. al. (2018) "Authenticity and Personal Creativity: How EarSketch Affects Student } \\
\text { Persistence. Proc. of the 49th ACM Technical Symposium on Computer Science Education. } \\
\text { Baltimore, MD, USA. }\end{array}$ \\
\hline TestMyCreativity & $\begin{array}{l}\text { Oihus, P. et al. (2013) "Design Wars: Developing student creativity through competition", Proc. } \\
\text { of the IEEE Frontiers in Education Conference, Oklahoma City, OK, USA. }\end{array}$ \\
\hline $\begin{array}{l}\text { Assessment Scale of Creative } \\
\text { Collaboration }\end{array}$ & $\begin{array}{l}\text { Romero, M. et al. (2018) "Co-creativity assessment in the process of game creation", Proc. of the } \\
12 \text { th European Conference on Game Based Learning, Sophia Antipolis, France. }\end{array}$ \\
\hline $\begin{array}{l}\text { RIBS - Runco Ideational Behavior } \\
\text { Scale }\end{array}$ & $\begin{array}{l}\text { Runco, M. A. et al. (2001) "Development and Psychometric Integrity of a Measure of Ideational } \\
\text { Behavior", Creativity Research Journal, 13(3-4), 393-400. }\end{array}$ \\
\hline $\begin{array}{l}\text { ECCI-i - Epstein Creativity } \\
\text { Competencies Inventory for } \\
\text { Individuals }\end{array}$ & $\begin{array}{l}\text { Shell, D. F. et al. (2013) "Associations of students' creativity, motivation, and self-regulation with } \\
\text { learning and achievement in college computer science courses", Proc. of the IEEE Frontiers in } \\
\text { Education Conference, Oklahoma City, OK, USA. }\end{array}$ \\
\hline $\begin{array}{l}\text { EDICOS - Emotion/motivation- } \\
\text { related Divergent and Convergent } \\
\text { Thinking Styles Scale }\end{array}$ & $\begin{array}{l}\text { Soroa G. et al. (2015) "Assessing interactions between cognition, emotion, and motivation in } \\
\text { creativity", Thinking Skills and Creativity, 17, 45-58. }\end{array}$ \\
\hline $\begin{array}{l}\text { IACEST - Indirect Assessment of } \\
\text { Creativity through the Estimation } \\
\text { of Stereotypical Thinking }\end{array}$ & $\begin{array}{l}\text { Susnea, I., Vasiliu, G. A (2016) "Fuzzy Logic Software Tool and a New Scale for the Assessment } \\
\text { of Creativity", International Journal of Computers Communications \& Control, 11(3), 441-449. }\end{array}$ \\
\hline
\end{tabular}

Muitos modelos possuem base teórica em trabalhos antecedentes ([Torrance e Goff 1989][Guilford 1967][Sternberg 1985]), ressaltando que a avaliação da criatividade e os 
fatores que a compõem já são há muito tempo analisados. Os fatores utilizados com mais frequência para avaliar a criatividade são originalidade, fluência, flexibilidade e curiosidade. De forma geral, observou-se uma falta de um consenso de como avaliar, e a própria terminologia/definição da criatividade, considerando sua base teórica. A maioria dos modelos utiliza a escala Likert, com quantidade de questões que varia de 18 a 60 . As aplicações relatadas se referem a amostras com tamanhos (n) variando entre 30 a 2270.

De forma geral, os modelos são propostos para diversos domínios. O único modelo encontrado que traz alguma associação entre criatividade e ensino de computação é o proposto por McKlin et al. (2018), avaliando alunos do Ensino Médio antes e depois de utilizarem uma ferramenta de aprendizado colaborativa para programação de músicas.

Assim, como resultado do mapeamento da literatura evidencia-se a falta de modelos de avaliação de criatividade na Educação Básica, especificamente para o contexto de ensino de computação.

\section{Metodologia de Pesquisa}

Foi adotada uma metodologia multi-método consistindo em três etapas. Na primeira etapa foi realizado um mapeamento sistemático da literatura visando levantar os modelos existentes e quais suas características. O mapeamento foi conduzido seguindo Petersen et al. (2015) e os resultados estão apresentados de forma resumida na seção 2.

Na segunda etapa, foi desenvolvido o modelo de avaliação da criatividade a partir das informações encontradas no mapeamento sistemático, seguindo os procedimentos para desenvolvimento de escalas [DeVellis 2016] e do guia para projeto de questionários [Kasunic 2005]. Seguindo a abordagem GQM [Basili et al. 1994], a habilidade criatividade foi decomposta em quatro dimensões (fluência, flexibilidade, originalidade e elaboração), conforme Guilford (1950) e acrescentou-se mais outras seis dimensões observadas na literatura. A partir dessa decomposição e de um conjunto de itens análogos aos obtidos da literatura, foram definidos os itens do questionário. Ponderou-se diversos aspectos como a similaridade e a redundância, de forma a agregar aspectos comuns, a adequá-los ao contexto brasileiro e a linguagem utilizada pelo público alvo. A versão preliminar do questionário foi analisada por um painel de especialistas das áreas de computação e pedagogia e representantes do público alvo. A partir do feedback, diversos itens tiveram sua formulação alterada para melhor compreensão, alguns itens foram removidos e outros foram separados em dois itens para melhor avaliação.

A terceira etapa visou avaliar o modelo SCORE como instrumento de medição da criatividade em termos de confiabilidade e validade. Para isso, foi realizada uma série de estudos de caso conforme proposto por Yin (2009). Nesses estudos, o instrumento SCORE foi aplicado na prática usando o design de pesquisa de uma única avaliação sem qualquer tipo de tratamento. Foram coletadas 76 respostas de alunos da Educação Básica de 5 escolas. Após a coleta de dados, esses foram reunidos em uma única amostra para análise. A avaliação da confiabilidade e da validade do construto foi realizada a partir da análise dos dados coletados, seguindo as definições de Trochim e Donnelly (2018) e o guia de desenvolvimento de escalas proposto por DeVellis (2016). A confiabilidade foi medida por meio do coeficiente alfa de Cronbach que mede a consistência interna do questionário. A validade do construto foi analisada usando o fator exploratório e baseando-se na evidência da validade convergente e discriminante obtida pelo cálculo das correlações de Spearman entre os itens. Os resultados 
da análise estatística foram interpretados pelos pesquisadores no contexto do ensino de computação, bem como, foram propostos aperfeiçoamentos no instrumento de medida.

\section{Desenvolvimento do Modelo de Avaliação SCORE}

Com base nos resultados do mapeamento da literatura, foi proposto o instrumento de autoavaliação denominado SCORE (aSsessing COmputing cREativity) que é apresentado na Tabela 2. SCORE objetiva a autoavaliação da criatividade dos estudantes no contexto do ensino da computação na Educação Básica.

\section{Tabela 2. Instrumento de autoavaliação da criatividade SCORE}

\begin{tabular}{|c|c|}
\hline Fator & Item \\
\hline $\begin{array}{c}\text { Amplia } \\
\text { conhecimentos e } \\
\text { habilidades }\end{array}$ & $\begin{array}{l}\text { 10. Eu gosto de aprender coisas novas. } \\
\text { 11. Não tenho medo de aprender coisas novas. } \\
\text { 12. Com os conhecimentos que tenho, consigo resolver um novo problema. } \\
\text { 13. Gosto de participar de atividades extracurriculares para aprender coisas novas (pesquisa de } \\
\text { campo, palestras, cursos). } \\
\text { 14. Eu entro na Internet várias vezes para aprender coisas novas. } \\
\text { 15. Eu gosto de discutir assuntos dando a minha opinião. } \\
\text { 16. Eu sei como aproveitar elogios e críticas ao refazer um trabalho da escola. } \\
\text { 17. Eu consigo fazer críticas construtivas. } \\
\text { 18. Eu aprendo com os meus erros. }\end{array}$ \\
\hline Conexão & $\begin{array}{l}\text { 19. Eu posso descobrir relações entre o uso de computadores e o seu impacto na sociedade. } \\
\text { 20. Eu consigo entender e interpretar o tipo do problema a ser resolvido, por exemplo, como fazer a } \\
\text { tarefa de matemática. } \\
\text { 21. Eu consigo prestar atenção e entender a ideia de outras pessoas. } \\
\text { 22. Consigo criar novas soluções combinando coisas que já conheço. }\end{array}$ \\
\hline Originalidade & $\begin{array}{l}\text { 31. Eu gosto de criar meus próprios jogos digitais. } \\
\text { 32. Eu tento resolver um problema sozinho antes de perguntar a alguém. } \\
\text { 33. Eu gosto de criar novos aplicativos para celular e não só usar os que já existem. } \\
\text { 34. Eu já fiz algo usando o computador que nunca pensei que fosse possível. } \\
\text { 35. Eu acho que é importante pensar em coisas de várias formas diferentes. } \\
\text { 36. Eu imagino muitas coisas que ainda não existem. } \\
\text { 37. Gosto de modificar programas de computador a partir de programas que outras pessoas } \\
\text { compartilharam. } \\
\text { 38. Tenho ideias de como fazer novos jogos e de como melhorá-los. }\end{array}$ \\
\hline
\end{tabular}




\begin{tabular}{|c|c|}
\hline Fluência & $\begin{array}{l}\text { 39. Eu consigo imaginar soluções diferentes para resolver um problema (por exemplo, como chegar } \\
\text { mais rápido à escola). } \\
\text { 40. Eu acho fácil escrever uma história para um jogo. } \\
\text { 41. Eu consigo escrever um programa de computador. } \\
\text { 42. Quando eu crescer, eu gostaria de trabalhar com algo que tivesse que pensar em várias ideias } \\
\text { novas. } \\
\text { 43. Eu consigo pensar numa lista de coisas que gastam pouco dinheiro e podem melhorar a minha } \\
\text { escola. } \\
\text { 44. Eu sou capaz de explicar um programa de computador para colegas. } \\
\text { 45. Tenho ideias de aplicativos para celular que eu poderia desenvolver. }\end{array}$ \\
\hline Flexibilidade & $\begin{array}{l}\text { 46. Sou capaz de combinar ideias de maneiras que outras pessoas não tentaram. } \\
\text { 47. Eu consigo pensar em novas formas de usar uma panela. } \\
\text { 48. Eu gosto de trabalhar criando coisas novas ao invés de fazer exercícios repetitivos. } \\
\text { 49. Consigo achar os materiais que preciso para desenvolver uma ideia. } \\
\text { 50. Se não há um certo recurso, tento achar uma solução com outros recursos disponíveis. }\end{array}$ \\
\hline Elaboração & $\begin{array}{l}\text { 51. Eu me importo com os detalhes quando faço alguma coisa. } \\
\text { 52. Eu presto atenção nas cores e fontes utilizadas na tela de um aplicativo de celular. } \\
\text { 53. Depois de usar um aplicativo interessante, eu gosto de conversar com alguém sobre ele. } \\
\text { 54. Quando estou interessado em alguma coisa, eu presto atenção em todos os detalhes. } \\
\text { 55. Quando faço algum trabalho de escola, gosto de deixá-lo bonito e enfeitado. } \\
\text { 56. Gosto de fazer de forma bonita as telas de jogos ou aplicativos de celular que crio. }\end{array}$ \\
\hline
\end{tabular}

A escala de resposta escolhida foi uma escala tipo Likert de 5-pontos de "Discordo totalmente" até "Concordo totalmente", seguindo a maioria dos modelos encontrados no mapeamento sistemático.

\section{Avaliação do Modelo SCORE}

Com o objetivo de avaliar a confiabilidade e a validade do instrumento de medição do modelo SCORE, ele foi aplicado em um estudo de caso no contexto na Educação Básica.

\subsection{Definição e Execução da Avaliação}

Para avaliar a confiabilidade e validade do questionário de autoavaliação foram analisadas as perguntas: (i) Há evidências de consistência interna no instrumento de medição? (ii) Há evidências de validade convergente e discriminante no instrumento de medição? Os dados para a análise foram coletados a partir da aplicação do questionário em um estudo de caso na Educação Básica (sem aplicação de tratamento). Os dados foram coletados por meio do preenchimento do questionário, disponibilizado de maneira online (devido a pandemia), por estudantes de diferentes turmas. O estudo foi aprovado pelo Comitê de Ética da Universidade Federal de Santa Catarina.

O questionário foi aplicado durante os meses de março e abril de 2020. Participaram da pesquisa um total de 76 estudantes da Educação Básica com idades de 8 a 17 anos de cinco escolas de Educação Básica (públicas e privadas) da grande Florianópolis/SC.

\subsection{Análise dos Dados}

Os dados coletados foram agrupados em uma única amostra, de modo a obter uma maior precisão e poder estatístico.

\section{Há evidências de consistência interna no instrumento de medição?}

O questionário em sua totalidade apresenta o coeficiente alfa de Cronbach de 0,961, sendo classificado como excelente em relação a sua consistência interna [Devellis 2016]. Foi também realizado o cálculo do coeficiente retirando cada item, caso esse fosse excluído 
IX Congresso Brasileiro de Informática na Educação (CBIE 2020)

Anais do XXXI Simpósio Brasileiro de Informática na Educação (SBIE 2020)

(Tabela 3). Essa análise busca identificar se algum item do instrumento de medição está prejudicando a confiabilidade do instrumento.

Tabela 3. Coeficiente alfa de Cronbach para cada item caso fosse excluído

\begin{tabular}{|l|c|l|c|l|c|}
\hline Item & $\begin{array}{l}\text { Alfa de Cronbach, se } \\
\text { item for excluído }\end{array}$ & Item & $\begin{array}{l}\text { Alfa de Cronbach, se } \\
\text { item for excluído }\end{array}$ & Item & $\begin{array}{l}\text { Alfa de Cronbach, se } \\
\text { item for excluído }\end{array}$ \\
\hline 1 & 0.960 & 20 & 0.960 & 39 & 0.959 \\
\hline 2 & 0.960 & 21 & 0.959 & 40 & 0.960 \\
\hline 3 & 0.960 & 22 & 0.960 & 41 & 0.960 \\
\hline 4 & 0.960 & 23 & 0.961 & 42 & 0.960 \\
\hline 5 & 0.960 & 24 & 0.961 & 43 & 0.960 \\
\hline 6 & 0.960 & 25 & 0.960 & 44 & 0.960 \\
\hline 7 & 0.960 & 26 & 0.960 & 45 & 0.960 \\
\hline 8 & 0.960 & 27 & 0.960 & 46 & 0.960 \\
\hline 9 & 0.961 & 28 & 0.961 & 47 & 0.960 \\
\hline 10 & 0.960 & 29 & 0.960 & 48 & 0.960 \\
\hline 11 & 0.960 & 30 & 0.961 & 49 & 0.960 \\
\hline 12 & 0.959 & 31 & 0.960 & 50 & 0.960 \\
\hline 13 & 0.959 & 32 & 0.960 & 51 & 0.960 \\
\hline 14 & 0.960 & 33 & 0.960 & 52 & 0.960 \\
\hline 15 & 0.960 & 34 & 0.960 & 53 & 0.960 \\
\hline 16 & 0.960 & 35 & 0.960 & 54 & 0.960 \\
\hline 17 & 0.960 & 36 & 0.960 & 56 & 0.960 \\
\hline 18 & 0.960 & 37 & 0.960 & & \\
\hline 19 & 0.959 & 38 & 0.960 & & \\
\hline
\end{tabular}

Os valores obtidos para o alfa de Cronbach de cada item variaram entre 0,959 e 0,961, o que indica que nenhum dos itens prejudica a consistência interna do instrumento de avaliação. Dessa forma, por esses critérios, não há nenhuma indicação para a exclusão de quaisquer itens.

\section{Há evidências de validade convergente e discriminante nos itens do instrumento de medição?}

A análise foi realizada por meio da obtenção de evidências de validade convergente e discriminante do instrumento, mediante ao cálculo de correlação dos itens. A validade convergente indica se itens que deveriam estar relacionados, por medir uma única dimensão, estão de fato relacionados. A validade discriminante indica se itens que não deveriam estar relacionados, de fato não estão relacionados [Trochim e Donnelly 2008]. Para tanto, foi utilizada a matriz de correlação não-paramétrica de Spearman e o cálculo do coeficiente de Cohen (1988). De acordo com Cohen (1988), uma correlação, em valores absolutos, entre itens é considerada moderada quando estiver entre 0,29 e 0,50 e alta quando for maior do que 0,50. Já a correlação em valores absolutos menor do que 0,29 indica que o item do questionário pode não estar medindo a dimensão pretendida.

Analisando a correlação entre os itens do questionário que medem cada habilidade, verificou-se que para a habilidade "Personalidade criativa e curiosidade" a maioria dos itens apresenta correlação moderada e alta. E um dos itens apresentou correlação divergente com outros três, indicando não medir o mesmo fator. Com relação a habilidade "Amplia habilidades e conhecimentos", em geral, os itens apresentaram correlação moderada e alta, indicando que medem o mesmo fator. Os itens da habilidade "Conexão" também demonstram bons resultados quanto à sua validade. Apenas dois dos itens apresentaram baixa correlação entre si. Já a habilidade "Ousadia" apresentou, de uma maneira geral, baixa correlação entre seus itens. Os itens da habilidade "Originalidade" obtiveram uma correlação moderada. Os itens da habilidade "Fluência" demonstraram bons resultados quanto à sua validade. A 
maioria dos pares possui correlação moderada a alta, sendo que apenas um dos itens não apresentou correlação significativa com os demais. Os itens da habilidade "Flexibilidade" apresentaram bons resultado com todos os itens possuindo correlação moderada. Apenas dois dos itens apresentaram muito baixa correlação entre si. Os itens da habilidade "Elaboração" apresentaram correlação moderada e alta entre seus itens.

Considerando o conjunto das habilidades e de seus itens correspondentes, a maioria apresentou correlação moderada e alta entre seus itens, como por exemplo as habilidades de "Originalidade" e "Fluência", o que indica boa correlação interna. Apenas três habilidades "Personalidade criativa e curiosidade", "Amplia habilidades e conhecimentos" e "Ousadia" tiveram alguns itens com correlação divergente.

\subsection{Ameaças a Validade}

Para reduzir ameaças quanto a confiabilidade, foi adotado um processo sistemático para o desenvolvimento do SCORE, incluindo uma revisão preliminar dos itens da avaliação feita por especialistas e representantes do público alvo, a fim de assegurar a consistência dos itens e clareza. A análise estatística dos dados foi embasada no guia de desenvolvimento de escalas de DeVellis (2016), e dessa forma, espera-se conter os impactos da escolha de um método de avaliação que não seja propício.

Quanto a ameaça referente à validade externa, considerando o tamanho da amostra limitado devido a pandemia de COVID-19, impossibilitando a aplicação do questionário de forma presencial nas escolas. Para contornar essa dificuldade, uma coleta online obtendo 76 respostas de alunos da Educação Básica foi realizada. Para verificar os resultados de forma mais ampla, foram coletados também respostas no ensino superior de 121 alunos. Como não foram observadas diferenças significativas na análise dessa amostra total, assume-se a validade dos resultados com a amostra da Educação Básica [Lehmkuhl 2020]. Porém, mesmo assim, recomenda-se a revisão dos resultados com maiores amostras de Educação Básica. Contudo, este resultado inicial demonstrou resultados satisfatórios quanto a sua confiabilidade e validade em relação a avaliação da criatividade.

\section{Conclusão}

Os resultados da análise de confiabilidade do instrumento mostraram uma excelente consistência interna dos itens, com um alfa de Cronbach igual a 0,961, mostrando que as respostas entre os itens são consistentes e precisas. A análise da validade do modelo foi realizada por meio da correlação entre os itens, em que também foram obtidos bons resultados. Assim, a avaliação do instrumento SCORE indica que ele é um instrumento válido para avaliar a criatividade e que pode ser aperfeiçoado numa futura versão. Considerando a importância de se dispor de instrumentos de avaliação da criatividade no contexto de computação na Educação Básica no Brasil, o instrumento SCORE, com livre acesso, pode ser um recurso para os professores e para as escolas na promoção da criatividade e possibilitar que a autopercepção dos estudantes sobre os aspectos relativos ao desenvolvimento dessa habilidade nas suas vidas.

\section{Agradecimentos}

Agradecemos a todos que participaram da avaliação do questionário. 
IX Congresso Brasileiro de Informática na Educação (CBIE 2020)

Anais do XXXI Simpósio Brasileiro de Informática na Educação (SBIE 2020)

Este trabalho foi apoiado pelo CNPq (Conselho Nacional de Desenvolvimento Científico e Tecnológico - www.cnpq.br), entidade do governo brasileiro voltada ao desenvolvimento científico e tecnológico.

\section{Referências}

Basili, V. et al. (1994) “Goal, Question Metric Paradigm”. Encyclopedia of Software Engineering, John Wiley \& Sons, 528-532.

Benett, V. E. et al. (2013) "Computing creativity: Divergence in computational thinking." Proc. of the 44th ACM Technical Symposium on Computer Science Education, 359-364.

Binkley, M. et al., (2011) "Defining 21st Century Skills". Draft white paper 1. Melbourne: The University of Melbourne, Melbourne, Australia.

Bordini, A. et al. (2017) "Pensamento Computacional nos Ensinos Fundamental e Médio". In Anais do Simpósio Brasileiro de Informática na Educação, Recife, Brasil.

Cavallo, D. et al. (2016) "Inovação e Criatividade na Educação Básica: Dos conceitos ao ecossistema”. Revista Brasileira de Informática na Educação, 24(2).

Cohen, J. (1988) "Statistical Power Analysis for the Behavioral Sciences" New York: Routledge Academic.

DeVellis, F. (2016) "Scale development: theory and applications", 4. ed. SAGE Publications, 280.

Fields, Z., Bisschof, C. (2013) “A Theoretical Model to Measure Creativity at a University”, Journal of Social Sciences, 34, 47-59.

Guilford, J. P. (1950) “Creativity”, American Psychologist. 5, 444-454.

Guilford, J. P. (1956) “The structure of intellect”. Psychological Bulletin, 53(4), 267-293.

Guilford, J. P. (1967) "Creativity: Yesterday, today and tomorrow, The Journal of Creative Behavior, 1, 3-14.

Henessey, B., Amabile, T. (1988) "Storytelling as a means of assessing creativity", Journal of Creative Behavior, 22, 235-247.

Jindal-Snape, D. et al. (2013) "The impact of creative learning environments on learners: A systematic literature review”, Improving Schools, 16(1), 21-31.

Kasunic, M. (2005) "Designing an effective survey. Handbook CMU/SEI-2005-HB-004, Software Engineering Institute. Carnegie Mellon University, Pittsburgh, USA.

Kaufman, J. C. (2019). "Self-assessments of creativity: Not ideal, but better than you think". Psychology of Aesthetics, Creativity, and the Arts, 13(2), 187-192.

Kupers, E. et al. (2018) "Creativity in the Here and Now: A Generic, Micro-Developmental Measure of Creativity", Frontiers in Psychology, 9, 2095.

Lai, E. R., Viering, M. (2012) “Assessing 21 st Century Skills: Integrating Research Finding”. In Proc. of the Nat. Council on Measurement in Education, Vancouver, Canadá.

Lehmkuhl, G. Desenvolvimento de um Modelo de Avaliação de Criatividade no Ensino da Computação na Educação Básica. Trabalho de Conclusão de Curso. (Graduação em Sistemas de Informação) - Universidade Federal de Santa Catarina. 
Mioto, F. et al. (2019) "bASES21 - Um Modelo para a Autoavaliação de Habilidades do Século XXI no Contexto do Ensino de Computação na Educação Básica”. Revista Brasileira de Informática na Educação, 27(1).

Nakano, T. C. (2020). "Métodos Atuais para Avaliação da Criatividade: Vantagens e Questionamentos". Avaliação Psicológica, 19(1), 97-105.

Pásztor, A. et al. (2015) “Technology-based assessment of creativity in educational context”, Thinking Skills and Creativity, 18, 32-42.

Petersen, K. et al. (2015) "Guidelines for conducting systematic mapping studies in software engineering: an update". Information and Software Technology, 64, 1-18.

Petty, G. (1997) “How to be better at creativity”, London: Kogan Page Limited.

Rahimi, H. et al. (2011) "Relationship between Knowledge Management Process and Creativity among Faculty Members in the University", Interdisciplinary Journal of Information, Knowledge, and Management, 6, 017-033.

Rhodes, M. (1961) “An analysis of creativity”, The Phi Delta Kappan, 42, 305-310.

Richardon, C., Mishra, P. (2018) "Learning environments that support student creativity: Developing the SCALE". Thinking Skills and Creativity, 27, 45-54.

Runco, M. A., Acar, S. (2012) "Divergent Thinking as an Indicator of Creative Potential", Creativity Research Journal, 24(1), 66-75.

Sousa-Filho, P. G. (2011) "Desenvolvimento da criatividade em Ambientes Digitais em Professores dos Anos Iniciais do Ensino Fundamental". Tese de doutorado, Universidade Federal do Rio Grande do Sul, Porto Alegre, Brasil.

Souza, A. C. D. et al. (2017) "Psychometric properties in instruments evaluation of reliability and validity", Epidemiologia e Serviços de Saúde, 26, 649-659.

Sternberg, R. (1985) “Implicit Theories of Intelligence, Creativity, and Wisdom”, Journal of Personality and Social Psychology, 49, 607-627.

Torrance, E. P. (1966) “The Torrance Tests of Creative Thinking”, Princeton: Personnel Press.

Torrance, E. P., Goff, K. (1989) “A quiet revolution”. Journal of Creative Behavior, 23(2), 136-145.

Trochim, M., Donnelly, P. (2008) "Research methods knowledge base", 3. ed., Mason: Atomic Dog Publishing.

Yin, R. K. (2009) "Case study research: design and methods" 4th. ed., Beverly Hills: Sage Publications. 